\title{
Rapid reduction in black carbon emissions from China: evidence from 2009-2019 observations on Fukue Island, Japan
}

Yugo Kanaya et al.

Correspondence to: Yugo Kanaya (yugo@ jamstec.go.jp)

The copyright of individual parts of the supplement might differ from the CC BY 4.0 License. 

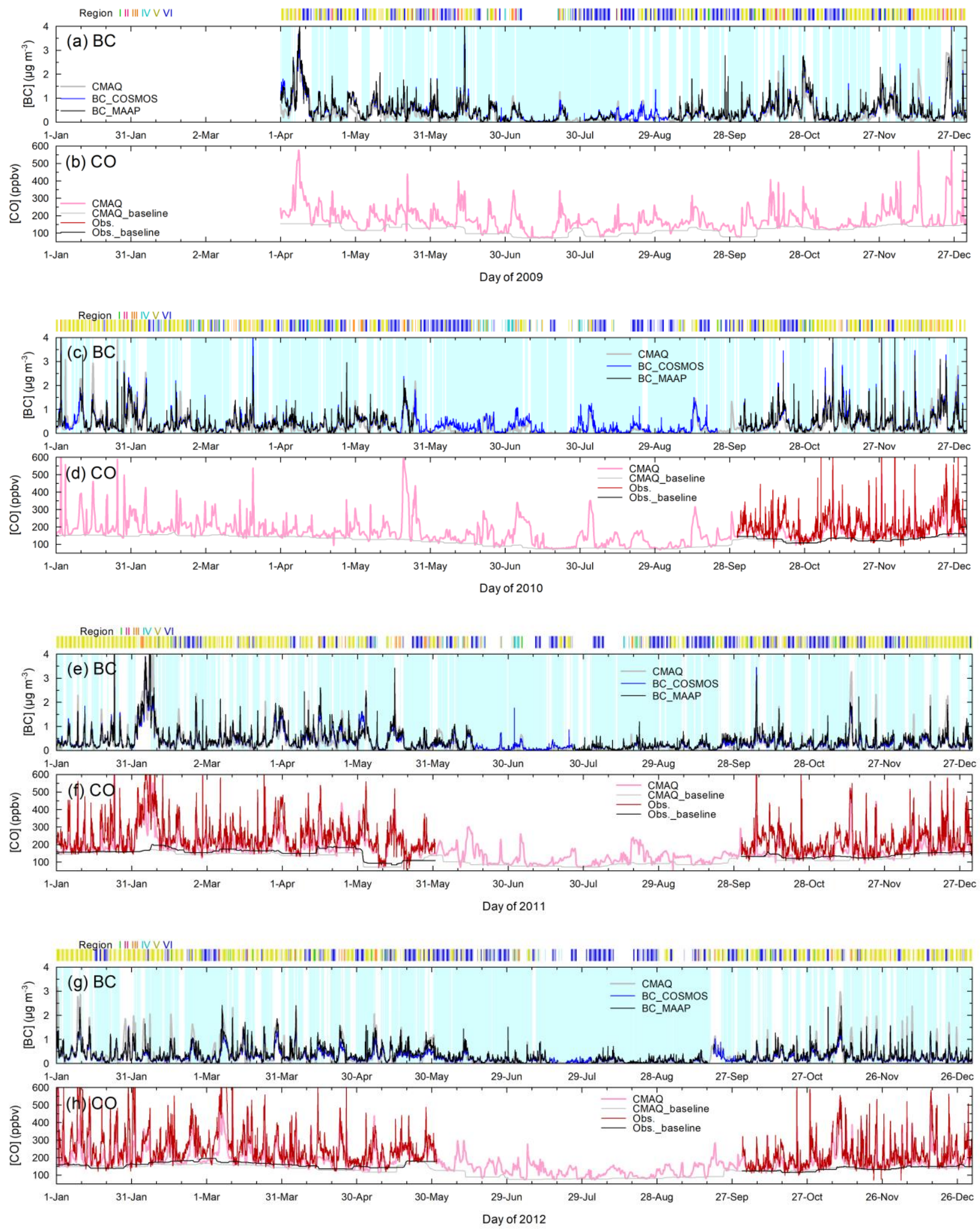

Figure S1. Black carbon (BC) mass concentrations and $\mathrm{CO}$ mixing ratios from observations and model simulations during individual years. In the top panel of each year, the air-mass origin areas are indicated with colored vertical bars. The light 5 blue bands indicate periods with Accumulated Precipitation along Trajectory (APT) $>1 \mathrm{~mm}$. 

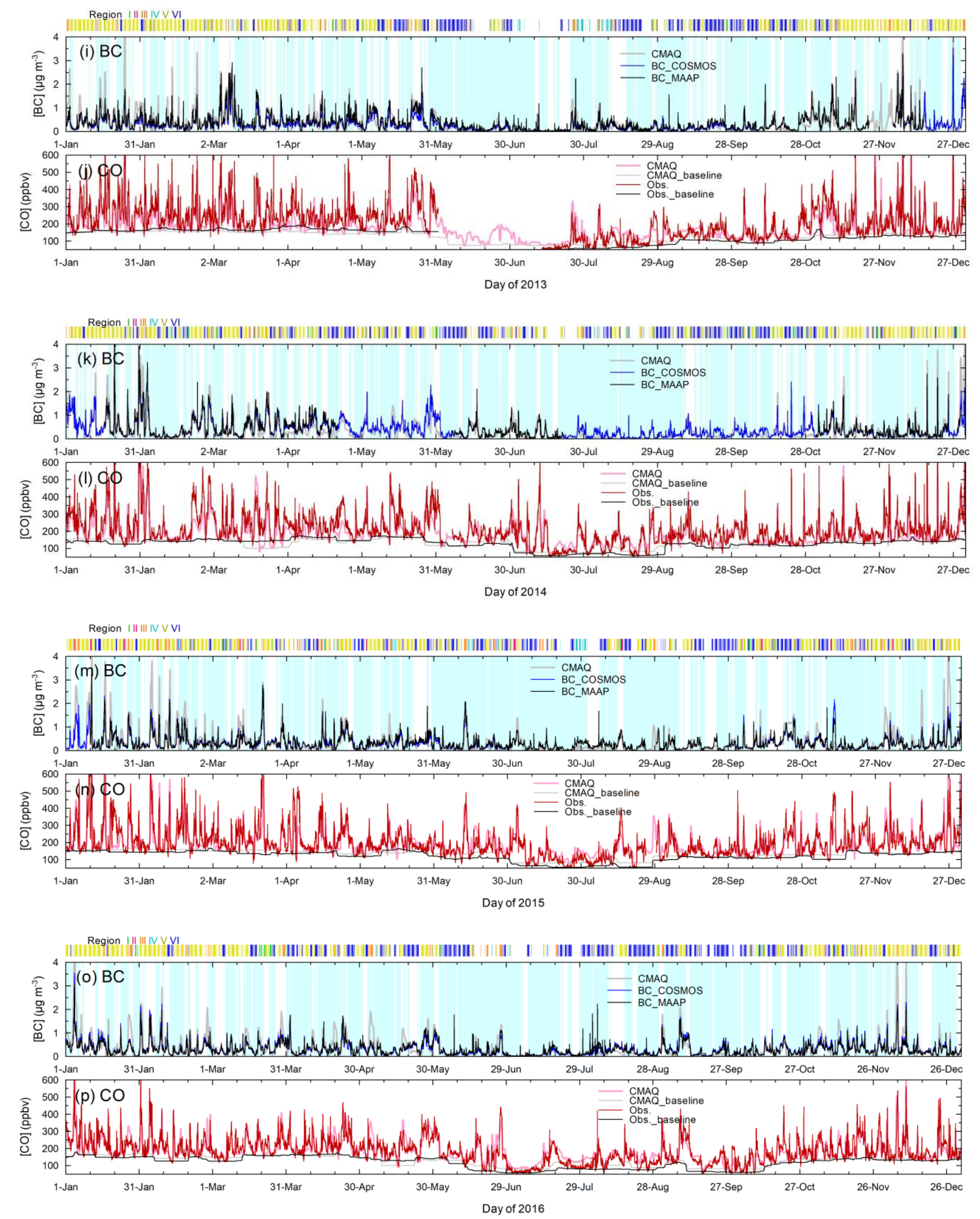

Figure S1. Continued. 
Region | || || IV V V|
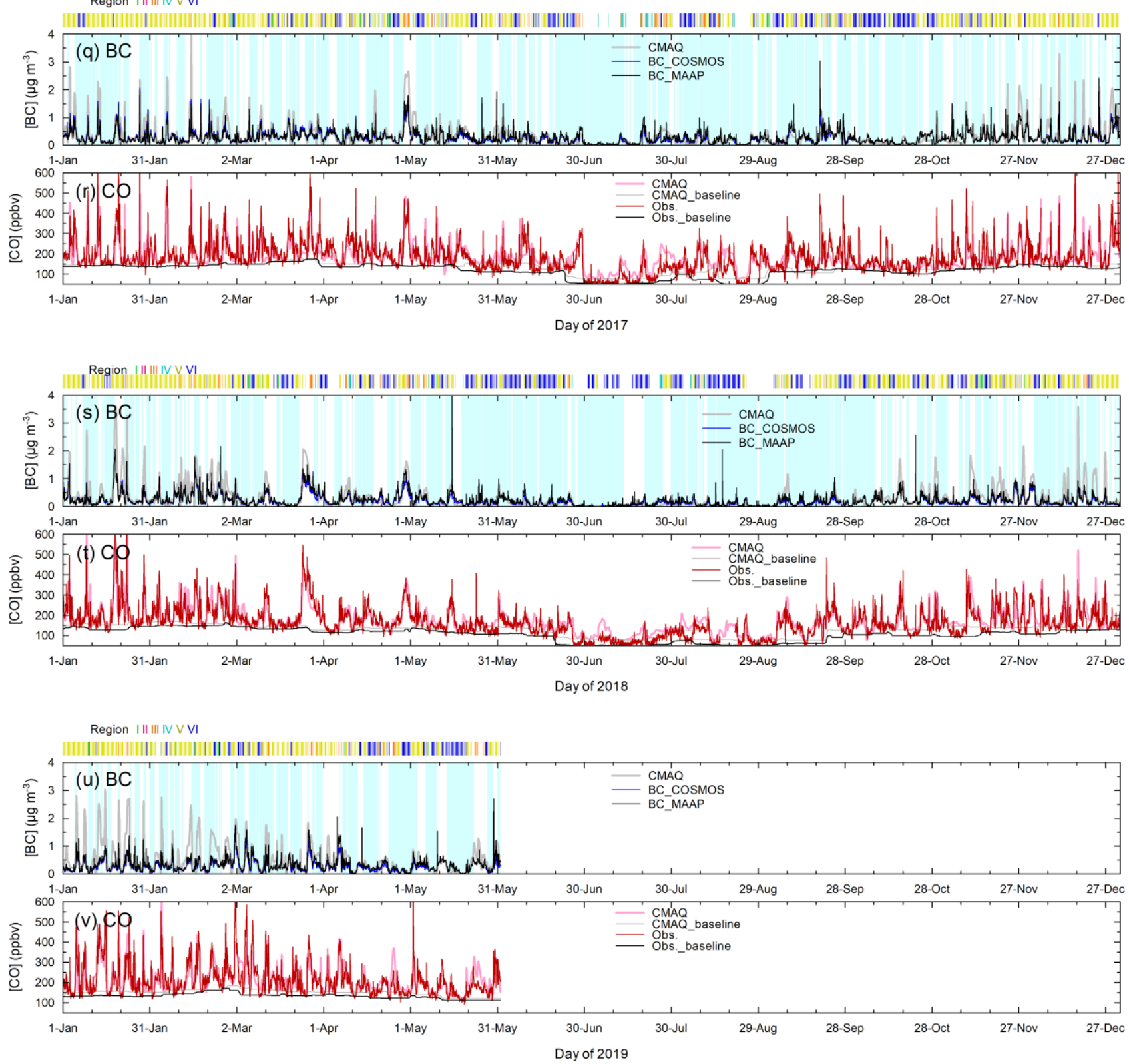

Figure S1. Continued. 


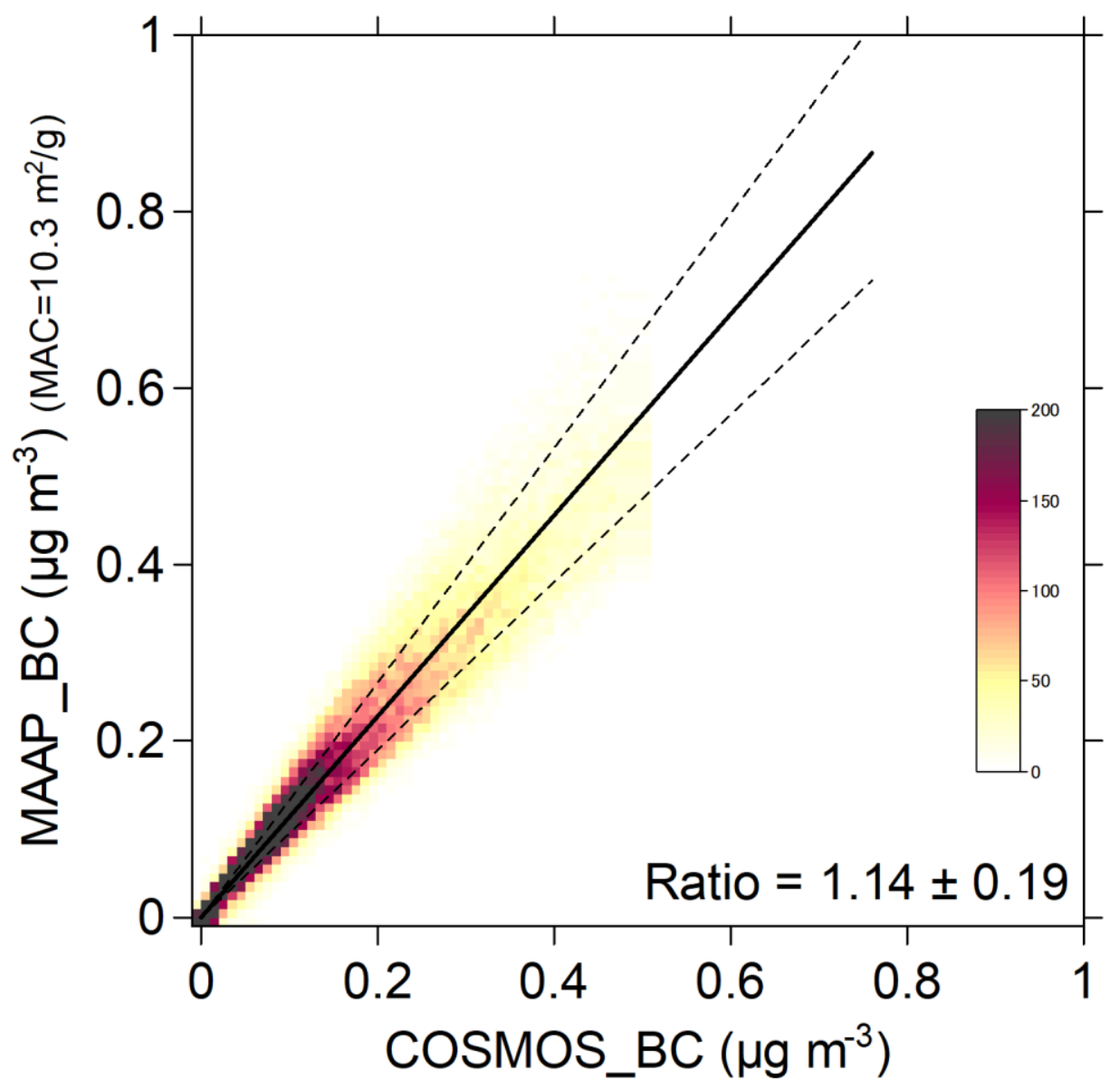

Figure S2. Correlation between hourly BC concentrations from COSMOS and MAAP instruments. The average and $1 \sigma$ range of the MAAP_BC/COSMOS_BC ratio $(1.14 \pm 0.19)$ are shown with solid and broken lines. 
u, $00 \mathrm{Z}, 925 \mathrm{hPa}$

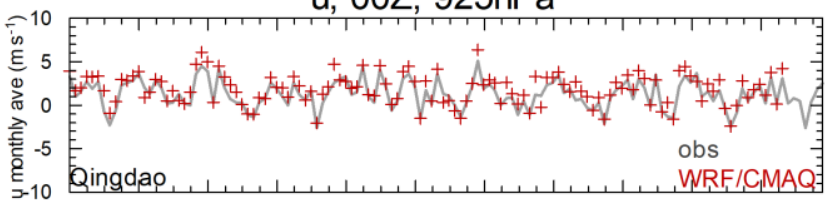

Jan-09 Jan-10 Jan-11 Jan-12 Jan-13 Jan-14 Jan-15 Jan-16 Jan-17 Jan-18 Jan-19

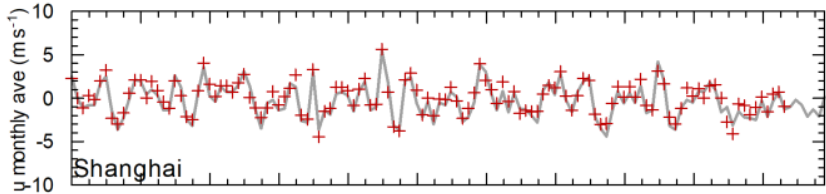

Jan-09 Jan-10 Jan-11 Jan-12 Jan-13 Jan-14 Jan-15 Jan-16 Jan-17 Jan-18 Jan-19

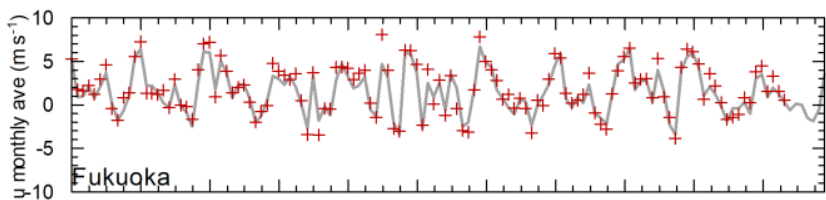

Jan-09 Jan-10 Jan-11 Jan-12 Jan-13 Jan-14 Jan-15 Jan-16 Jan-17 Jan-18 Jan-19

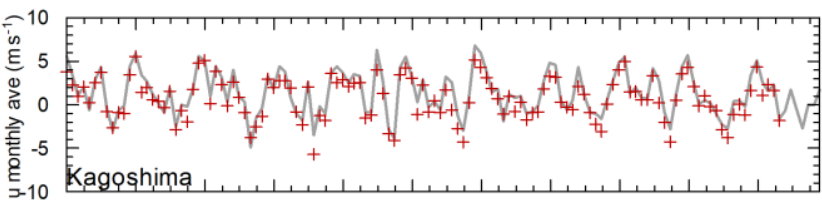

Jan-09 Jan-10 Jan-11 Jan-12 Jan-13 Jan-14 Jan-15 Jan-16 Jan-17 Jan-18 Jan-19

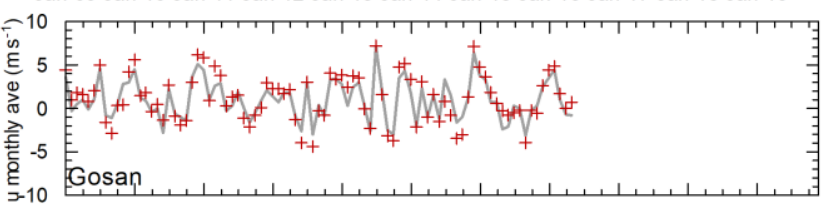

Jan-09 Jan-10 Jan-11 Jan-12 Jan-13 Jan-14 Jan-15 Jan-16 Jan-17 Jan-18 Jan-19 v, 00Z, $925 \mathrm{hPa}$

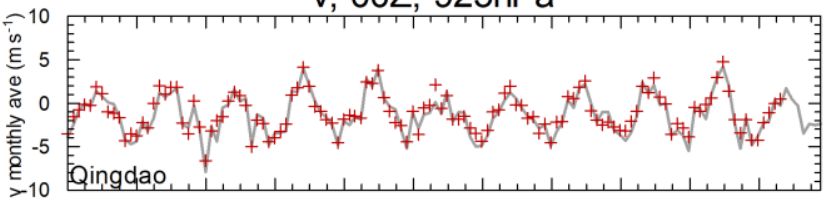

Jan-09 Jan-10 Jan-11 Jan-12 Jan-13 Jan-14 Jan-15 Jan-16 Jan-17 Jan-18 Jan-19

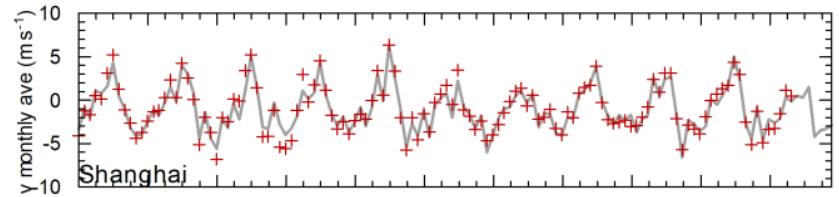

Jan-09 Jan-10 Jan-11 Jan-12 Jan-13 Jan-14 Jan-15 Jan-16 Jan-17 Jan-18 Jan-19

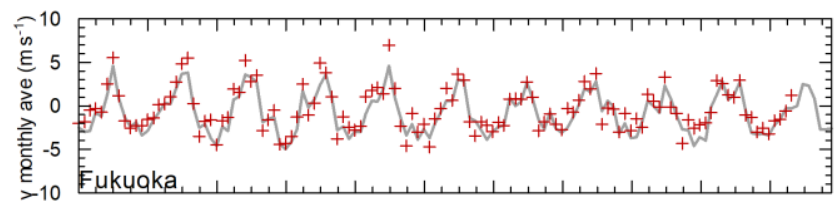

Jan-09 Jan-10 Jan-11 Jan-12 Jan-13 Jan-14 Jan-15 Jan-16 Jan-17 Jan-18 Jan-19

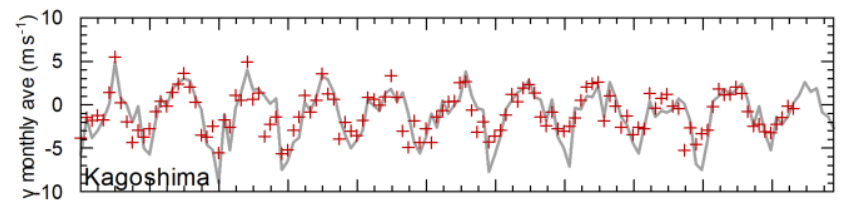

Jan-09 Jan-10 Jan-11 Jan-12 Jan-13 Jan-14 Jan-15 Jan-16 Jan-17 Jan-18 Jan-19

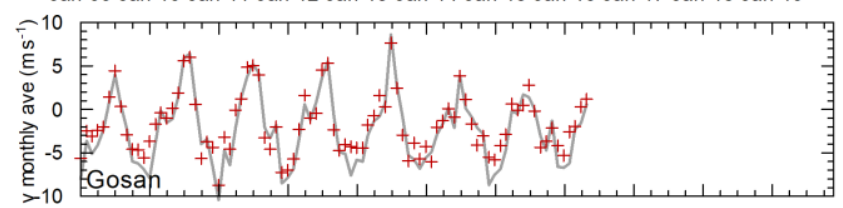

Jan-09 Jan-10 Jan-11 Jan-12 Jan-13 Jan-14 Jan-15 Jan-16 Jan-17 Jan-18 Jan-19

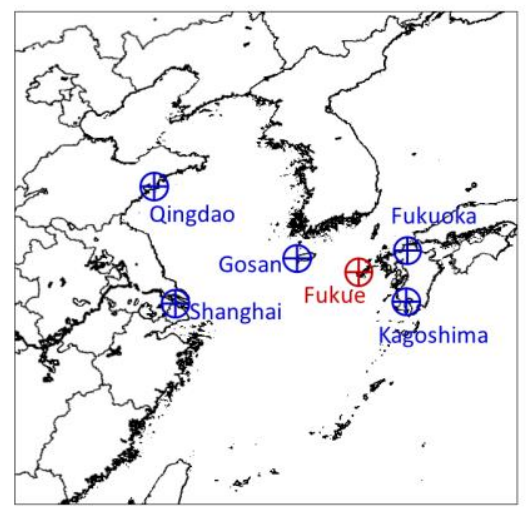

Figure S3. Observed monthly-averaged zonal (u) and meridional (v) wind speeds at $925 \mathrm{hPa}$ at $00 \mathrm{Z}$ over five locations near Fukue (grey lines) were compared with those at corresponding girds of WRF/CMAQ model simulations. 


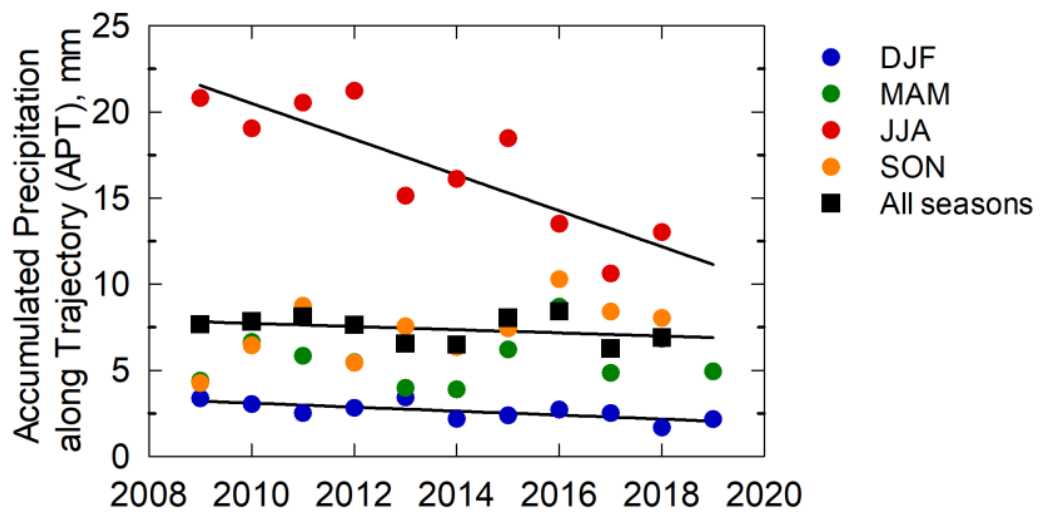

Figure S4. Trends in APT for air masses arriving at Fukue for all and individual seasons. Regression lines are shown for JJA, all seasons, and DJF. 

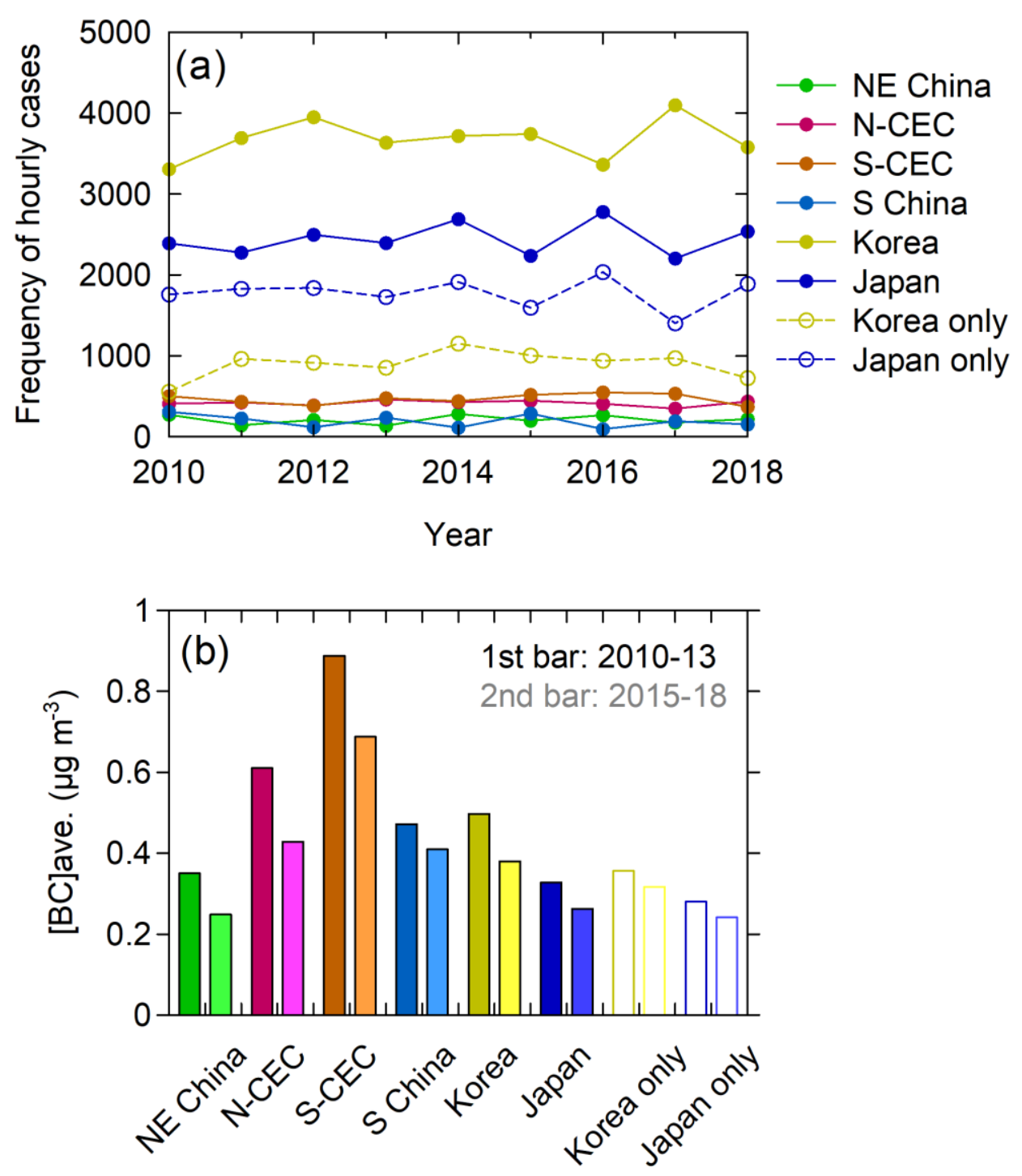

Figure S5. (a) Statistics and trends of number of hourly cases of observed air masses from different origin areas. (b) Averaged BC mass concentrations for individual air mass origin areas, for the periods of 2010-13 and 2015-18. 


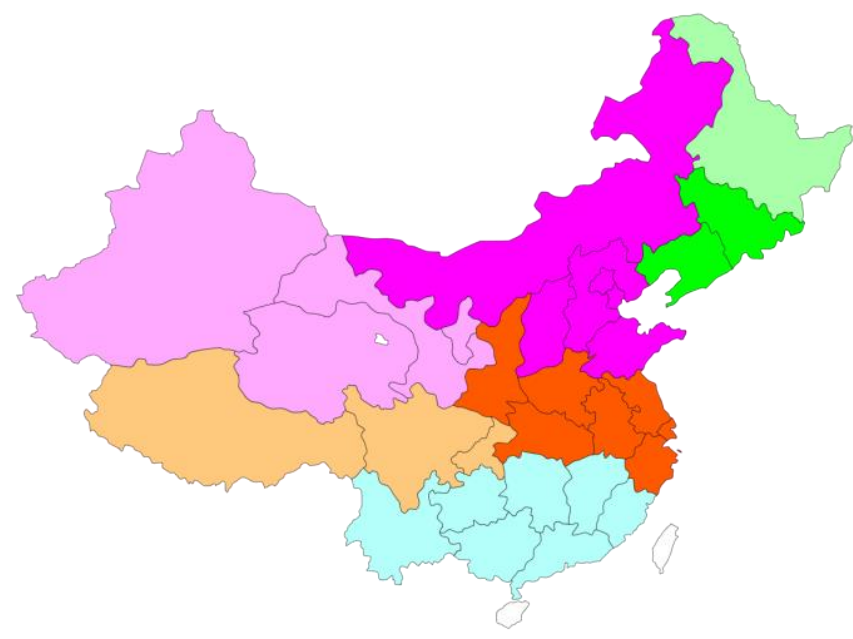

Figure S6. Approximate assignment of Chinese provinces into the four regions of China: Region I (NE China) as green, Region II (N-CEC) as magenta, Region III (S-CEC) as orange, and Region IV (S China) as light blue. The pale colors indicate provinces with reduced signal weight and therefore $\mathrm{BC}$ emissions from such provinces were assumed unchanged in 5 a case study. 


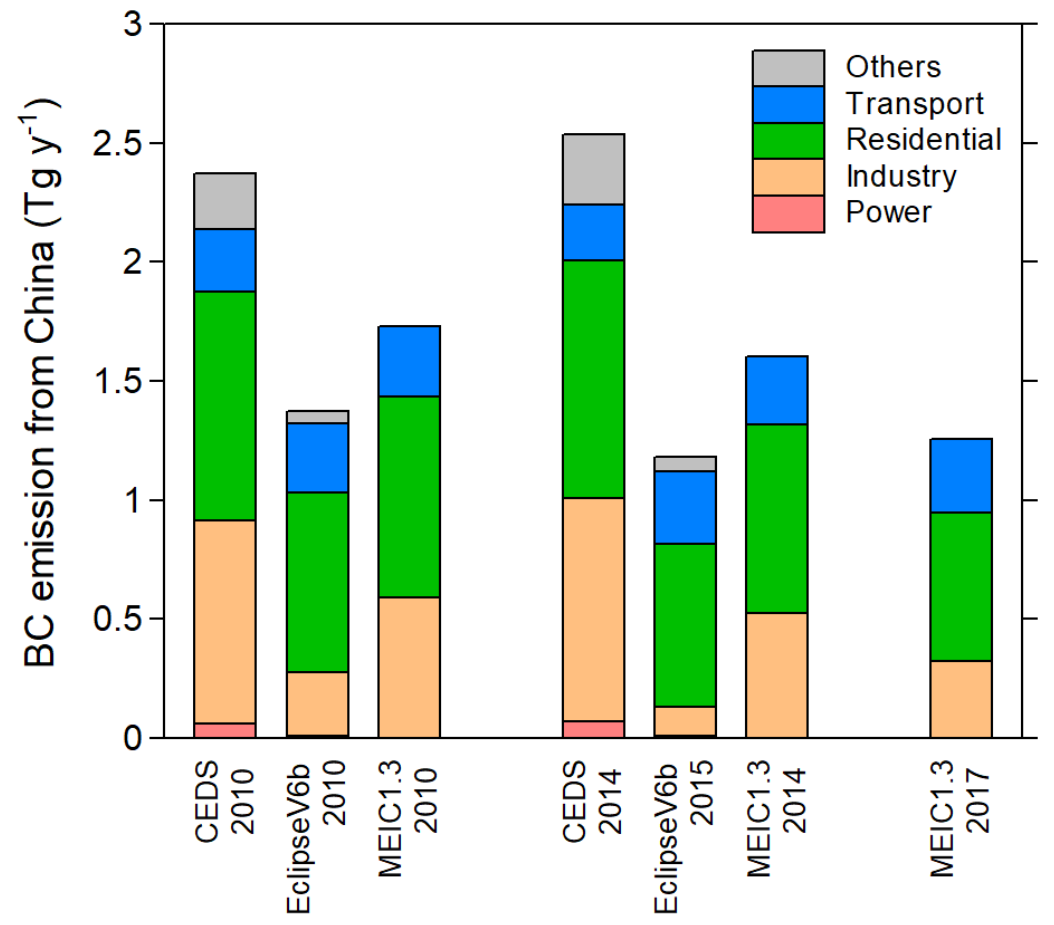

Figure S7. Sectoral breakdown of BC emission from China for CEDS, Eclipse V6b, and MEIC1.3 for 2010, 2014 (2015 for Eclipse V6b), and 2017 (for MEIC1.3 only). CEDS, Eclipse V6b, and MEIC1.3 data are from Hoesly et al. (2018), Klimont et al. (in prep., 2019), and Zheng et al. (2018), respectively. 
Table S1. Trends of estimated emission of CO for all seasons and DJF.

\begin{tabular}{|c|c|c|c|c|c|c|}
\hline \multicolumn{7}{|c|}{ All seasons } \\
\hline & $N$ & $\mathrm{E}(2014-17) / \mathrm{REAS} 2.1(2008)$ & Trend $\left(\mathrm{y}^{-1}\right)$ & Trend $\left(\% \mathrm{y}^{-1}\right)$ & $R$ & $P$ \\
\hline All regions I-VI & 1962 & 1.31 & $-0.07 \pm 0.01$ & $-5.2 \pm 0.9$ & -0.97 & 0.029 \\
\hline Region I (NE China) & 1201 & 1.40 & $-0.07 \pm 0.03$ & $-5.3 \pm 1.9$ & -0.89 & 0.11 \\
\hline Region II (N-CEC) & 2444 & 1.17 & $-0.04 \pm 0.01$ & $-3.7 \pm 0.8$ & -0.95 & 0.047 \\
\hline Region III (S-CEC) & 2810 & 1.03 & $-0.07 \pm 0.02$ & $-6.9 \pm 1.5$ & -0.96 & 0.043 \\
\hline Region IV (S China) & 1014 & 1.05 & $-0.10 \pm 0.02$ & $-9.6 \pm 2.0$ & -0.96 & 0.041 \\
\hline Region V' (Korea only) & 5345 & 1.45 & $-0.04 \pm 0.02$ & $-2.5 \pm 1.5$ & -0.77 & 0.23 \\
\hline Region VI' (Japan only) & 9304 & 1.35 & $-0.05 \pm 0.00$ & $-3.8 \pm 0.4$ & -0.99 & 0.0087 \\
\hline \multicolumn{7}{|c|}{ DJF } \\
\hline & $N$ & $\mathrm{E}(2012-18) / \mathrm{REAS} 2.1(2008)$ & Trend $\left(\mathrm{y}^{-1}\right)$ & Trend $\left(\% \mathrm{y}^{-1}\right)$ & $R$ & $P$ \\
\hline All regions I-VI & 17884 & 1.46 & $-0.11 \pm 0.01$ & $-7.3 \pm 0.6$ & -0.98 & $8.0 \times 10^{-5}$ \\
\hline Region I (NE China) & 398 & 1.63 & $0.05 \pm 0.02$ & $2.8 \pm 1.3$ & 0.69 & 0.085 \\
\hline Region II (N-CEC) & 927 & 1.20 & $-0.06 \pm 0.01$ & $-4.9 \pm 0.7$ & -0.95 & 0.00089 \\
\hline Region III (S-CEC) & 914 & 1.07 & $-0.11 \pm 0.02$ & $-10.1 \pm 1.8$ & -0.93 & 0.024 \\
\hline Region V' (Korea only) & 2034 & 1.94 & $-0.10 \pm 0.04$ & $-5.4 \pm 1.9$ & -0.78 & 0.038 \\
\hline Region VI' (Japan only) & 789 & 1.78 & $-0.09 \pm 0.02$ & $-5.0 \pm 1.1$ & -0.90 & 0.006 \\
\hline
\end{tabular}

